Conclusions There was reasonable homogeneity using 25-50 clusters per module (representing $3-15 \%$ of the number of jobs per questionnaire), but important heterogeneity remained. A more efficient use of expert judgment may be to assess exposure at the cluster-level and then, within expert-identified heterogeneous clusters, at the job-level.

\section{VALIDATION OF QUESTIONNAIRE ITEMS AMONG DUTCH CONSTRUCTION WORKERS USING DIRECT WORKPLACE OBSERVATIONS}

${ }^{1} \mathrm{H}$ Zilaout, ${ }^{2}$ Timmerman, ${ }^{2}$ Heederik, ${ }^{3}$ Spee, ${ }^{2}$ Smit. ${ }^{1}$ University Utrecht/ Institute for Risk Assessment Sciences, Utrecht, Nederland; ${ }^{2}$ Institute for Risk Assessment Sciences (IRAS), Utrecht, Nederland; ${ }^{3}$ Arbouw, Harderwijk, Nederland

10.1136/oemed-2013-101717.290

Objectives Contact dermatitis is widely present among construction workers. The risk of developing occupational contact dermatitis among this group is probably related to occupational exposure to chemicals. In addition, frequency of glove use and exposure to water during hand washing may also influence the prevalence of hand eczema. The aim of this project was to validate questionnaire items on hand dirtiness and glove use by comparing with direct workplace observations.

Methods A cross-sectional study was conducted at 13 different construction sites in the Netherlands. The questionnaire covered general information such as age, gender, occupation and specific questions regarding hand conditions, glove use, glove types, glove replacement, frequency of hand washing and possible symptoms of hand eczema during the last 12 months.

Data of 177 participants (95\% response rate) were analysed. Agreement between observation and questionnaire was assessed by calculating Cohen's kappa. In addition, the sensitivity and specificity were determined. Multivariate analysis was conducted to assess the association between hand eczema and workplace determinants.

Results Observation of hand dirtiness, glove use and glove types were found to agree well with questionnaires, with kappa's of $0.75,0.61$ and 0.88 respectively. The 1-year prevalence of hand eczema was $45.8 \%$. Multivariate logistic regression analysis with hand eczema as dependent variable showed a statistically significant correlation with 'hand cream use' (PR 2.4 (95\% CI: 1.6 to 3.8)) and 'hand washing efforts' (PR 1.5 (95\% CI: 1.1 to 2.0)). There was also a significant positive association between hand eczema and 'hand dirtiness' and 'glove use'.

Conclusions There is a strong correlation found between direct observations and questionnaire. Therefore, it is reasonable to consider that these questionnaire items are suitable to be used in future epidemiological studies. Hand eczema was often reported and was positively associated with potential determinants of exposure asked for in the questionnaire.

\section{OCCUPATIONAL EXPOSURE TO ISOCYANATES; A BASELINE EXPOSURE ASSESSMENT AS BASIS FOR AN INTERVENTION STRATEGY}

E H A M van Deurssen. IRAS, Utrecht, Nederland

10.1136/oemed-2013-101717.291

Objectives Occupational exposure to isocyanates has been associated with the development of occupational asthma. This study serves as baseline measurement within an intervention study, aimed at 1) conducting a detailed exposure assessment and determine exposure determinants, 2) providing input for the development of a broad intervention strategy, and 3) evaluating the effectiveness of respiratory protection.

Methods Personal task-based inhalation samples for mixing, spraying and gun cleaning were collected among 37 workers, divided over eighteen companies. Relevant information regarding potential exposure determinants and behavioural and organisational factors was obtained by performing a walk through survey and a questionnaire. Mixed effect regression models were used to identify associations between exposure and work practices, behavioural factors (e.g. knowledge, awareness), and organisational factors (e.g. support towards OSH-programs). The level of respiratory protection during workplace activities was assessed among 22 workers.

Results Spray painting results in the highest exposure levels compared (47 $\mu \mathrm{g} / \mathrm{m}^{3} \mathrm{NCO}$ ) to mixing and gun cleaning (respectively $0.15 \mu \mathrm{g} / \mathrm{m}^{3} \mathrm{NCO}$ and $0.7 \mu \mathrm{g} / \mathrm{m}^{3} \mathrm{NCO}$ ). Worker orientation and spray location seem to be indicative for exposure. A full overview of our analyses and a first outline of the intervention strategy will be presented during the conference. The use of respirators seems task-dependent, where first analyses seem to indicate that the protection factor is above $95 \%$.

Discussion Although we found decreased exposure levels compared to earlier studies, we still see possibilities for interventions to further decrease exposure. For instance through the organisation of work, the frequency of (proper) use of control measures, and further improvements in the use of respirators. The results of our exposure assessment will be used to perform health impact assessment, presented in another abstract.

\section{Session: 14. Exposure assessment II}

\section{ASSESSMENT OF PSYCHOSOCIAL EXPOSURE: HOW TO ESCAPE THE TRIVIALITY TRAP?}

1) P B Bonde, ${ }^{2}$ Gullander, ${ }^{3}$ Grynderup, ${ }^{3}$ Willert, ${ }^{4}$ Hansen, ${ }^{5}$ Høgh, ${ }^{6}$ Persson, ${ }^{7}$ Thomsen, ${ }^{8}$ Mors, ${ }^{9}$ Rugulies, ${ }^{3}$ Kolstad. ${ }^{1}$ Bispebjerg Hospital, Copenhagen, Denmark; ${ }^{2}$ Department of Occupational and Environmental medicine, Bispebjerg Hospital, Copenhagen, Denmark; ${ }^{3}$ Department of Occupational Medicine, Danish Ramazzini Centre, Aarhus University, Aarhus, Denmark; ${ }^{4}$ Department of Public Health, Copenhagen University, Denmark, Copenhagen, Denmark; ${ }^{5}$ Department of Psychology, Copenhagen University, Denmark, Copenhagen, Denmark; ${ }^{6}$ The National Research Centre for the Working Environment, Copenhagen, Denmark, Copenhagen, Denmark; 'Department of Occupational and Environmental medicine, Bispebjerg Hospital, Copenhagen, Denmark; ${ }^{8}$ Aarhus University Hospital, Risskov, Aarhus, Denmark; ${ }^{9}$ The National Research Centre for the Working Environment, Copenhagen, Denmark

\subsection{6/oemed-2013-101717.292}

Objectives Workplace bullying may be a strong determinant of major depression, but only a few studies provide prospective data and none provide independent information on bullying. In a follow-up study we analysed newly-onset depression in relation to workplace bullying measured at the individual level (perceived bullying) and at the work-unit level (witnesses reporting bullying).

Methods Danish employees were recruited from two Danish cohorts of 3.743 and 2.617 workers, respectively. Cohort members received a questionnaire at baseline in 2006-07 with twowave follow-ups in 2008-09 and 2011. Workplace bullying was measured by self-labelling and by the proportion of employees in a work unit who had witnessed workplace bullying "now and 
again" to "daily" over the past 6 months. For the latter purpose all participants were identified with their work-unit (471 work units, number of employees ranging between 1 and 161). The work-units were grouped according to the proportion of employees. who had witnessed workplace bullying within their work units. New cases of depression were diagnosed at the end of two-year follow-up periods using Schedules for Clinical Assessment in Neuropsychiatry (SCAN) interviews and the Major Depression Inventory questionnaire.

Results During the follow-up period, we identified 177 new cases of depression. The odds ratio for newly-onset depression among participants reporting bullying occasionally was 1.62 [95\% CI 0.95-2.77] and among those reporting bullying often it was 5.73 [95\% CI 2.37-13.90]. The risk of newly-onset depression by percentage of employees witnessing bullying in work-units was for 1-20\%: 0.83 [95\% CI 0.48-1.43], 2130\%: 0.87 [95\% CI $0.49-1.55$ ], and >30\%: 1.08 [95\% CI 0.61-1.90].

Conclusions Self-reported frequent bullying predicts development of depression but a work environment defined by witnesses of bullying does not. These findings have implications for the understanding of workplace bullying and options for preventive actions.

\section{SEX DIFFERENCES IN MUSCULAR LOAD AMONG HOUSE PAINTERS PERFORMING IDENTICAL WORK TASKS}

${ }^{1} \mathrm{~T}$ H H Heilskov-Hansen, ${ }^{2}$ Meyland, ${ }^{3}$ Alkjær, ${ }^{3}$ Koblauch, ${ }^{2}$ Mikkelsen, ${ }^{4}$ Wulff Svendsen, ${ }^{2}$ Frølund Thomsen, ${ }^{5}$ Hansson, ${ }^{3}$ Bruun Simonsen. 'Bispebjerg University Hospital, Copenhagen NV, Denmark; '2Department of Occupational and Environmental Medicine, Bispebjerg University Hos, Copenhagen, Denmark; ${ }^{3}$ Department of Neuroscience and Pharmacology, University of Copenhagen, Copenhagen, Denmark; ${ }^{4}$ Danish Ramazzini Centre, University Department of Occupational Medicine, Herning, Herning, Denmark; ${ }^{5}$ Occupational and Environmental Medicine, Lund University, and University and Reg, Lund, Sweden

\subsection{6/oemed-2013-101717.293}

Objective The aim of the present study was to estimate possible differences in upper body muscular load between male and female house painters performing identical work tasks. Sexrelated differences in muscular activity may help explain why female house painters, and females in general, have more musculoskeletal complaints and disorders than men do.

Methods In a laboratory-setting, 16 male and 16 female house painters performed nine standardised work tasks common to house painters. Unilateral EMG recordings were obtained from the supraspinatus muscle by intramuscular electrodes and from the trapezius-, extensor- and flexor carpi radialis muscles by surface electrodes. Maximum voluntary contractions were performed, and both relative muscular load in $\% \mathrm{EMG}_{\max }$ as well as exerted force in Newton were assessed. APDF curves were obtained for each subject, and load distributions were characterised by the $10^{\text {th }}, 50^{\text {th }}$ and $90^{\text {th }}$ percentiles; sex differences were tested using a mixed model approach.

Results Women were exposed to a significantly $(\mathrm{P}=0.05)$ higher relative muscular load than men in the supraspinatus and forearm muscles in all tasks. Men exerted significantly $(\mathrm{P}=$ $0.05)$ more absolute force in the trapezius muscle at the $50^{\text {th }}$ percentile in all tasks, and in a single task also at the $10^{\text {th }}$ percentile. The differences between men and women were independent of tasks.

Conclusion Female house painters had a higher relative muscular load than their male colleagues, even though the men exerted more absolute force compared to the women. The effects of a higher relative muscular load, accumulated over years of work, may in part explain why musculoskeletal complaints and disorders in the upper body occurs more frequently among female than male house painters.

\section{DOES DEPRESSION SEVERITY PREDICT DIFFERENCES BETWEEN INDIVIDUAL AND WORK-UNIT AVERAGED MEASURES OF THE PSYCHOSOCIAL WORK ENVIRONMENT?}

${ }^{1} \mathrm{M}$ W Willert, ${ }^{2}$ Persson, ${ }^{2}$ Hansen, ${ }^{3}$ Mors, ${ }^{4}$ Thomsen, ${ }^{4}$ Bonde, ${ }^{5}$ Kolstad. ${ }^{1}$ Aarhus University Hospital, Aarhus, Denmark; ${ }^{2}$ The National Research Centre for the Working Environment, Copenhagen, Denmark; 'Aarhus University Hospital, Risskov, Aarhus, Denmark; ${ }^{4}$ Department of Occupational and Environmental Medicine, Bispebjerg Hospital, Copenhagen, Denmark; ${ }^{5}$ Department of Occupational Medicine, Danish Ramazzini Centre, Aarhus University Hospital, Aarhus, Denmark

\subsection{6/oemed-2013-101717.294}

Objectives It is debated if results from epidemiological studies on psychosocial factors at work and risk of depression are affected by the common rater problem, thus inflating associations by introducing reporting bias. Because depression is associated with cognitive distortions it is expected that severity of depression affects the level of reported exposures. The present study aims to investigate the possible dose-effect relationship between diagnosed severity of depression and differences between individual and work-unit averaged measures of the psychosocial work environment in a large epidemiological study. Methods In 20074291 Danish public employees within 378 different work units were enrolled in the study. Mean levels of psychological demands were computed for each work unit. Screening for depressive symptoms (SCL-DEP6) 329 persons scoring above cut-off were invited to a diagnostic interview (SCAN), resulting in 40 cases with mild, 43 with moderate, and 17 with severe depression, and 229 cases with no diagnosis of depression. Differences were analysed with ANOVA statistics.

Results For quantitative demands the mean score difference was 0.08 points for participants with mild depression compared to their work-unit average, 0.28 points for participants with moderate depression and 0.36 points for participants with severe depression $(\mathrm{p}=0.40)$.

Conclusions The data indicate a dose-effect pattern between severity of depression and divergence from work-unit averages. However, the results are not statistically significant and our initial hypothesis is not supported. Future perspectives involve including two follow-up waves to gather more cases and provide additional statistical power, and also allow adjustment for relevant confounders and changes in reporting over time. These results have implications regarding the need for exposure measures that are independent of reports from participants diagnosed with depression, but may also be explored as indicators of social marginalisation as a mechanism relevant to the development or maintenance of depression.

\section{ERGONOMIC EXPOSURE ASSESSED BY PRODUCTION STATISTICS}

${ }^{1} \mathrm{C}$ B Brauer, 'Bern, ${ }^{2}$ Alkjaer, 'Bonde, ${ }^{3}$ Helweg-Larsen, ${ }^{2}$ Koblauch, ${ }^{3}$ Moller, ${ }^{2}$ Simonsen, ${ }^{1}$ Thomsen, ${ }^{3}$ Thygesen, ${ }^{1}$ Mikkelsen. ${ }^{1}$ Department of Occupational and Environmental Medicine, Bispebjerg University Hospital, Copenhagen NV, Denmark; ${ }^{2}$ Department of Neuroscience and Pharmacology, University of Copenhagen, Copenhagen, Denmark; 